\title{
Simple Science, Quality Science
}

\author{
John R. Percy \\ Department of Astronomy, Erindale Campus, University of Toronto, \\ Mississauga ON, Canada L5L 1C6. e-mail: jpercy@erin.utoronto.ca
}

\begin{abstract}
This paper examines ways in which those in astronomicallydeveloping countries can begin to take part in meaningful astronomical research. It is based on a discussion paper, prepared by the author for a special session of IAU Commission 46 on 12 August 2000, and on the many comments contributed by the audience after the paper.
\end{abstract}

How can those in the astronomically-developing countries (ADCs) begin to take part in meaningful astronomical research? In this context, research is defined as "producing useful astronomical data or results, leading to presentations at conferences, and publications in journals by the observers, or by others". The answer to the question will differ from one ADC to another. It will depend on the local economic, structural, and human capacity. Possible answers should be the result of careful, ongoing discussions between the local astronomical community, and those from "outside" who wish to help. These discussions, and the follow-up assistance and advice, may have to be continued over 3-5 years, to overcome the initial "learning curve", and to build up the necessary momentum to reach the goal. Good research cannot begin instantly; there may have to be a transition period between "no research" and "front-line research". The IAU Commission 46 Program Groups on Advance Development, and on Teaching for Astronomical Development, are appropriate bodies to engage in these discussions.

It is assumed that the local researchers will begin with small astronomical telescopes - as small as 20-cm aperture. They may then progress to telescopes in the $40-\mathrm{cm}$ to $60-\mathrm{cm}$ range. The capabilities of small telescopes have recently (Irion 2000) been discussed in Science - one of the two most prestigious general science journals in the world. The government of Japan has very generously donated telescopes, of approximately 0.5 -m aperture, to several ADC's, and has provided training in Japan for some of the local astronomers. In addition to the research (and education) capabilities of such telescopes, they can serve as "attractors" of students, and they can give credibility to the local astronomy activities in the eyes of other academics, administrators, and government.

The issue of "quality science" is important. The quality can usually be assessed by the local observers in some way, but it is also useful to have an external mentor or collaborator who can assess the quality of the data being produced, and offer advice and instruction on how to improve. This is one 
advantage of participating in an international research collaboration. It takes a number of weeks or months to be sure that the equipment and instruments are working satisfactorily, and producing good data. Snowden (2001) has questioned whether astronomy research can be done in an ADC without extensive direction from "outside". It is true that some of the local astronomers must have a sense of how science works, and what research is all about. It is especially important for research to be part of the local university teaching curriculum, so that students will learn that knowledge is developed through research, not through textbooks. They must learn - preferably from experience - that research is exciting, and fun! Education also serves to heighten the profile of astronomy in the ADC, both within the universities, and among politicians and the public. There are many practical considerations to beginning astronomical research, and Kochhar (1996) has mentioned four:

- When a new astronomical facility is set up, it should be at a level consistent with the workshop facilities and infrastructural support available. The equipment should not overwhelm the user.

- For the initial training of manpower, co-operation should preferably be sought from countries which are culturally akin to the host country.

- Attempts should be made to integrate astronomical facilities with the teaching program.

- For best results, observational programs should be chosen so as to form a part of an international campaign.

Because of the small number of potential researchers in the ADCs, it would be most efficient and effective if the research was carried out collaboratively by several members of the local "astronomical community" - professional astronomers (if any), scientists and technologists from related disciplines (physics, engineering, math, statistics, geology), advanced amateur astronomers, undergraduate and graduate students, science teachers etc. Each of these groups brings special skills and enthusiasm to the research enterprise. On the other hand, there may be problems of hierarchy - such as lack of respect for the absolutely essential role that technicians play in making the equipment work effectively, or for the capabilities of students as researchers.

There is also the practical matter that astronomical research is usually done at night. That may raise issues of security, of accommodating mixed observing groups, or simply the tradition of "normal working hours".

Small telescopes can also be used for teaching at the high-school and university level, for public education, for obtaining images which can be used by the local media etc. These applications will not be discussed here, but they should certainly be integrated into the program of the telescope as a way to build up public support for astronomy.

The most obvious field of research (in part because it is my own) is the measurement of variable stars. This can be carried out at various levels of expense, technical difficulty, and sophistication: visual, photographic, photoelectric (PEP) and charge-coupled device (CCD) photometry. These require 
increasing levels of instrumentation for data acquisition, reduction, and analysis. All are useful for research. There are several organizations which support and co-ordinate such work - notably the American Association of Variable Star Observers (AAVSO). The demand for AAVSO visual observations, from the professional astronomical community, for instance, has increased by a factor of 20 since the 1970s. The AAVSO also has an education project Hands-On Astrophysics which provides a basic introduction to variable star observing and analysis, suitable for senior high school students, undergraduates, and amateur astronomers.

At a more advanced level, using a telescope of $20-\mathrm{cm}$ aperture or larger, researchers can do PEP or CCD photometry. The cost of a PEP photometer is about U.S. $\$ 1500$; a computer is useful but not absolutely essential. The cost of a CCD photometer is probably U.S. $\$ 5000$, and a computer (486 or better) is essential. The AAVSO co-ordinates both PEP and CCD photometry, and there is also the International Amateur-Professional Photoelectric Photometry (IAPPP) organization which supports such work internationally. At a slightly higher level, there is the international Center for Backyard Astrophysics (CBA), co-ordinated by Professor Joseph Patterson of Columbia University. The CBA uses an international network of CCD-equipped small telescopes to measure the rapid variations in cataclysmic variables. This has resulted in a steady stream of important papers. The typical cost of a CBA "station" is U.S. $\$ 12,000$ for a telescope, CCD camera, and computer. (This, of course, is a large amount of money for an ADC, but it is the kind of sum which might be provided by an external grant or donation. The goal is to make the best possible use of such funds.) There is a wide variety of other photometric projects which could be done through existing international collaborations: such as monitoring variable Be stars, RS CVn binaries, and other types of stars (Percy 1998).

Another interesting possibility in variable-star research is the analysis and interpretation of on-line archival data - from the HIPPARCOS epoch photometry database of 120,000 stars, for instance. HIPPARCOS maintains an education web page, and there is software available on the web (from the AAVSO, for instance) which can be used for the analysis.

There are other fields of astronomy which lend themselves to "simple, quality science". They are traditionally associated with advanced amateur astronomers, because the instrumental and technical requirements are modest (Percy \& Wilson 2000). [For those who are unconvinced of the value of amateur science, I recommend the eloquent article in Science by Mims (1999)] The fields include:

- Monitoring sunspots and sudden ionospheric disturbances, both caused by solar activity; both are co-ordinated by the AAVSO. The results are used by US government agencies as one measure of solar activity.

- Timing of occultations of stars by the Moon or other solar system objects; this is co-ordinated by the International Occultation Timing Association. Since the events are geographically specific, astronomers in the ADCs can provide useful results by timing events in their vicinities. This work provides information on the precise positions and motions of stars, and on 
their duplicity, and (in the case of grazing occultations) on the profile of the lunar limb.

- Meteor observing; this is co-ordinated by the American Meteor Organization, and other groups. Recent observations of the Leonid meteor shower by astronomers in Jordan and Morocco have illustrated the potential of such work. It can provide important information about the structure of the cometary debris which causes meteor showers.

There are additional, more challenging fields in which small telescopes with CCD or video-cameras are being used. These fields are opening up because of the availability of good small telescopes, CCD cameras, and powerful computers, at "affordable" (for amateur astronomers in the industrialized countries) prices - typically at least U.S. $\$ 10,000$ in total. Paul Boltwood, an amateur astronomer in climatically-underprivileged Ottawa, Canada, recently reached visual magnitude 23 with a $40-\mathrm{cm}$ telescope (the same level achieved by the Palomar 5-m telescope a generation ago). Ron Dantowitz, in Boston USA, has obtained ultra-high resolution images of the planets with a video-camera on a small telescope. These technologies have opened up fields of research such as (i) searching for optical after-glows from gamma-ray bursters; (ii) discovering and measuring faint asteroids and Kuiper Belt Objects, both photometrically and astrometrically; (iii) discovering and measuring distant supernovae; (iv) monitoring the photometric variations of Active Galactic Nuclei, etc.

Spectroscopy is also a suitable field for small telescopes. Simple, inexpensive spectrographs are available, using either photographic or CCD cameras, and they can be used for long-term monitoring of variable emission-line stars, for instance. Very few large, professional observatories carry out these kinds of observations. For further ideas, see Hearnshaw \& Cottrell (1986), Percy \& Wilson (2000), and many articles in Sky 8 Telescope.

Infrastructures are being set up to facilitate and co-ordinate partnerships between amateur and professional astronomers - the American Astronomical Society's (AAS) Working Group on Professional-Amateur Co-operation, for instance, and a series of conferences and scientific sessions (see Sky \& Telescope, June 2000).

All of these projects, of course, require training -- whether for astronomers from developed countries, or ADCs. This may be accomplished by having the trainees work with an experienced astronomer at an established observatory for as much time as is necessary, or by having the experienced astronomer spend some time with the astronomers in the ADCs. It may also be accomplished through hands-on workshops - as long as these are true workshops, and not just series of lectures. Hands-on workshops may be one of the projects to be organized by the AAS Working Group mentioned above. The UN Office of Basic Space Science, or the IAU, or organizations such as the AAVSO, might be willing and able to organize such workshops.

Again, there are practical problems. It may be difficult to find an experienced astronomer who is willing to spend several weeks in an ADC. The cost of bringing an astronomer from an ADC to an established observatory, or to a lengthy workshop, is not trivial. IAU Commission 46's Program Group on the 
Exchange of Astronomers (formerly Commission 38) must address these problems. How can workshops be funded?

There are other possibilities for research, which are not as directly linked with amateur astronomers. It is possible that an astronomer in an ADC has developed a line of research already, or has a collaboration with a professional astronomer elsewhere, which could be continued. The possibilities are endless; data could be provided from an archive, or by a collaborator; major observatories might be willing to provide opportunities for an astronomer in an ADC to work at the telescope. The research might involve a solar telescope, or a radio telescope, or it may involve computer simulation or theory. In general, young astronomers need opportunities to travel, and be immersed in a research atmosphere. They need opportunities to return to positions in the ADC's, and to continue their research. International travel cannot always be limited to senior astronomers from ADC's, who may no longer be active in research because of the pressure of administrative and education duties. Young astronomers are the hope of the future. The international development of astronomical research needs the help of all IAU members who might be willing to include the ADC's in their research programs. It needs the help of organizations such as the AAVSO and its counterparts in other countries and in other fields of astronomy. It needs the help of astronomical observatories and institutes, both large and small. I hope that these proceedings will open the eyes of individuals and organizations to the research needs and opportunities of the astronomically-developing countries.

\section{References}

Hearnshaw, J.B. \& Cottrell, P.L. 1986, Instrumentation and Research Programmes for Small Telescopes, D. Reidel, Dordrecht, The Netherlands.

Irion, R. 2000, it Science, 289, 7 July, 32.

Kochhar, R. 1996, paper presented at the Sixth UN/ESA Workshop on Basic Space Science, Bonn, Germany.

Mims, F.M. 1999, Science, 284, 2 April, 55.

Percy, J.R. 1998, Astrophys. Space Science, 258, 357.

Percy, J.R. \& Wilson, J.B. (editors) 2000, Amateur-Professional Partnerships in Astronomical Rsearch and Education, ASP Conf. Series, in press.

Snowden, M.S. 2001, this volume, pp. 266-275. 